\title{
A EVOLUÇão dA GEODÉSIA: DA OBSERVAÇÃo ÀS ESTRELAS AOS SATÉLITES
}

\author{
Sonia Maria Alves Costaํㅜ Luiz Paulo Souto Fortes ${ }^{2}$ \\ ${ }^{1}$ Instituto Brasileiro de Geografia e Estatística - IBGE; ${ }^{2}$ Universidade do Estado do Rio de Janeiro
}

$\mathrm{D}$

a mesma forma que as demais áreas das Geociências, a Geodésia passou por grandes transformações devido à evolução tecnológica ocorrida nos últimos 50 anos. Voltando um pouco no tempo, o objetivo principal da Geodésia consistia na determinação de uma posição na superfície terrestre, através das coordenadas - latitude, longitude e altitude. Estas coordenadas eram obtidas através de técnicas e métodos que proporcionassem a melhor precisão possível. Entretanto, dadas a grande evolução nas missões espaciais dedicadas ao melhor conhecimento do nosso planeta e as interações entre os seus diversos sistemas, é possível hoje monitorar também as variações que ocorrem nas coordenadas ao longo do tempo. Estas informações são de extrema importância para os sistemas de alerta de terremotos e erupções vulcânicas que estão sendo implantados em várias partes do mundo.

Este trabalho tem por objetivo relatar as transformações que a Geodésia do IBGE passou desde a sua primeira responsabilidade na campanha de determinação das coordenadas das sedes municipais, visando à atualização da Carta Geográfica do Brasil para o Censo de 1940, até os dias de hoje, com a adoção do Sistema de Referência Geocêntrico para as Américas SIRGAS2000. São abordadas também as mudanças que ocorreram na forma de atuar como gestor do Sistema Geodésico Brasileiro - SGB, através da disponibilização de serviços de posicionamento no portal do IBGE, os quais contam com um número expressivo de usuários, possibilitando inclusive o posicionamento em tempo real. 


\section{Uma campanha de levantamentos de coordenadas geográficas - Censo 1940 e as primeiras atividades geodésicas no IBGE}

O Conselho Nacional de Geografia (CNG) foi incluído no plano do trabalho do Recenseamento Geral da República de 1940 e recebeu a tarefa de atualizar a Carta Geográfica do Brasil, ao milionésimo, na determinação das coordenadas das sedes municipais brasileiras. A campanha foi iniciada por 10 engenheiros de diferentes instituições públicas dos estados, os quais foram instruídos e aparelhados com esta finalidade. Os levantamentos de precisão realizados na época baseavam-se em observações astronômicas e em determinações barométricas de altitudes. Esta atividade foi de grande sucesso e relevância para o CNG, pois conseguiu em pouco mais de 2 anos a determinação das coordenadas de cerca de 900 localidades.

Foi somente em março de 1944 que a Divisão de Cartografia do CNG iniciou os levantamentos de triangulação com equipamentos que pertenciam ao Observatório Nacional e à antiga Escola Politécnica do Rio de Janeiro, com o apoio logístico do Departamento Geográfico de Minas Gerais (DGMG). A primeira base geodésica foi medida nas proximidades de Goiânia com fio de invar - basímetro de Carpentier. No mesmo ano, por solicitação do Departamento Nacional de Produção Mineral do Ministério da Agricultura, esse primeiro grupo do CNG transferiu-se para Criciúma, em Santa Catarina, área da zona carbonífera catarinense em intensa atividade durante a $2^{\mathrm{a}}$ Guerra Mundial. A Base geodésica de Criciúma foi a primeira a ser medida com fita de invar, cedida ao CNG pelo U.S. Coast \& Geodetic Survey. Em Criciúma também foi medido o primeiro ponto astronômico de $1^{\mathrm{a}}$ ordem, utilizando-se uma luneta Bamberg. Ainda em Santa Catarina, surge em 1946 o segundo núcleo geodésico e são iniciadas as atividades do nivelamento geométrico de precisão.

Inicialmente foi projetada uma cadeia de triangulação ao longo do meridiano $49^{\circ}$ entre Goiânia e o sul do Brasil, que tinha como um de seus objetivos a vinculação das triangulações esparsas que já existiam em alguns estados, mas que apresentavam diferenças nas coordenadas dos vértices de ligação e consequentemente estas diferenças refletiam-se no mapeamento. Até 1954, conforme consta em um trabalho apresentado por Allyrio Hugueney de Mattos, no Congresso Brasileiro de Geografia, haviam sido estabelecidos 400 vértices de triangulação de $1^{\mathrm{a}}$ ordem (assim considerados), com 22 pontos de Laplace e 15 bases geodésicas. O nivelamento geométrico já possuía 3300 referências de nível, estabelecidas por meio de circuitos fechados entre os estados do Rio Grande do Sul e Rio de Janeiro. Tanto as medições dos ângulos horizontais, quanto dos pontos de Laplace, eram realizadas à noite e a produção dependia das condições meteorológicas. Outra questão também problemática na triangulação era a intervisibilidade entre as estações, havendo a necessidade da implantação das estações nos locais mais altos da região. 0 transporte dos equipamentos para estes pontos altos era realizado por animais e o tempo de permanência de uma equipe no campo era de muitos meses. Em algumas situações, como, por exemplo, no caso de vegetação alta e densa, havia a necessidade de construir torres que podiam chegar a até 30 metros. Estas torres possuíam duas estruturas, uma interna para apoiar o equipamento e outra externa para o operador.

Fazendo um cálculo aproximado destes primeiros 10 anos oficiais de levantamentos geodésicos, foram estabelecidos anualmente 40 vértices de triangulação e 330 referências de nível e 
ainda não se havia chegado nas regiões norte, nordeste e grande parte do centro-oeste. O cálculo geodésico era realizado manualmente em livros de cálculo por dois técnicos especializados, dada a sua complexidade. Resumidamente, o tempo decorrido entre o reconhecimento de uma região e o cálculo final das coordenadas poderia chegar a 5 anos ou mais.

Assim, sucintamente, tiveram início os trabalhos geodésicos no antigo CNG. Da década de 40 até os dias de hoje vai uma grande distância e um constante aprimoramento, à medida que se acompanha o desenvolvimento tecnológico, o que será comtemplado nos próximos itens desta nota.

\section{A Era do Posicionamento por satélites}

Poucos sabem que o posicionamento ou o georreferenciamento por satélites é usado no IBGE desde a década de 70, com o surgimento dos rastreadores de satélites artificiais. Foi com esta tecnologia que o Sistema Geodésico Brasileiro foi estendido à Região Amazônica, área até então não atingida face à dificuldade da realização de levantamentos geodésicos através dos procedimentos clássicos.

Como consequência do avanço das técnicas de posicionamento, as redes geodésicas passaram a ter um caráter tridimensional, marcada como a era da Geodésia Espacial (tridimensional), iniciada pelo sistema TRANSIT ou NNSS (Navy Navigation Satellite System) e substituído desde 1991 pelos Sistemas Globais de Navegação por Satélites, em inglês GNSS - Global Navigation Satellite Systems, nome genérico dado aos sistemas de navegação por satélite, dentre os quais destacam-se GPS, GLONASS, Galileo e BeiDou, sendo o GPS o pioneiro de todos eles e considerado operacional desde 1993. Os receptores GNSS utilizados na produção da geoinformação nas atividades da Geodésia e Cartografia da Diretoria de Geociências (DGC) proporcionam coordenadas com precisão de milímetros a centímetros, diferentemente daquele georrefenciamento praticado pelas pesquisas estatísticas da DPE, com o uso de dispositivos móveis (smartphones) que fornecem erros de alguns metros.

No caso dos posicionamentos de alta precisão, o uso dos sistemas GNSS causou uma verdadeira revolução nas atividades da Geodésia, sendo o responsável pela melhoria na qualidade da determinação de coordenadas em pelo menos duas ordens de magnitude. Antes, com o uso dos métodos clássicos, especialmente triangulação e poligonação, alcançava-se em geral precisões planimétricas relativas da ordem de 10 partes por milhão (ppm), enquanto que com os sistemas GNSS passou a ser possível obter precisões da ordem de 0,01 ppm ou melhores.

\section{Estações Geodésicas Ativas x Passivas}


Na aplicação dos métodos de posicionamento GNSS de precisão, adota-se geralmente o posicionamento relativo a partir do uso da fase da onda portadora, envolvendo a ocupação simultânea de estações com coordenadas conhecidas e das estações que se deseja determinar, motivando uma reflexão sobre as propriedades que as redes geodésicas deveriam possuir após o advento destes sistemas. O resultado desta reflexão foi o surgimento de redes geodésicas, denominadas "ativas", compostas por estações com coordenadas determinadas com alta precisão e dotadas de equipamentos GNSS geodésicos de operação contínua, facilitando assim o trabalho do usuário em tempo e custo.

Durante alguns anos, os dois tipos de redes geodésicas, ativa e passiva, foram estabelecidos concomitantemente, principalmente para atender a Lei 10.267/01, estabelecida pelo Instituto Nacional de Colonização e Reforma Agrária - INCRA, cuja proposta é o georreferenciamento de todas as propriedades rurais existentes no país, tendo como referência o SGB.

As redes ativas também possibilitaram o monitoramento contínuo das coordenadas das estações da rede que, considerando-se o seu nível milimétrico de precisão, passaram a revelar informações valiosas sobre a geodinâmica do local onde estão instaladas. Este aspecto viabilizou o desenvolvimento prático da Geodésia de quatro dimensões, sendo a quarta componente justamente o tempo de referência das coordenadas. Deste modo, a Geodésia é vista hoje como a ciência que se ocupa não apenas da precisa medição e representação de três propriedades fundamentais da Terra (sua forma geométrica, sua orientação no espaço e seu campo de gravidade), mas também das mudanças destas propriedades com o tempo. A evolução tecnológica, especialmente após o surgimento dos sistemas GNSS e das missões gravimétricas por satélites, tem permitido a avaliação precisa das alterações de coordenadas e gravidade ao longo do tempo, ampliando o escopo de atuação da Geodésia, considerada hoje fundamental para monitoramento das mudanças enfrentadas pelo planeta.

\section{O Estabelecimento e Expansão da Rede Brasileira de Monitoramento Contínuo dos Sistemas GNSS - RBMC}

Face às vantagens e importância das redes geodésicas ativas no contexto atual e através da colaboração com algumas iniciativas internacionais, o IBGE iniciou a capacitação no estabelecimento das estações ativas. A primeira estação, Fortaleza (FORT), foi estabelecida no INPE de Euzébio em 1993 através de uma colaboração com o National Geodetic Survey (NGS) dos Estados Unidos. A segunda estação foi a de Brasília (BRAZ) estabelecida em 1995 na Reserva Ecológica do Roncador, em colaboração com o Jet Propulsion Laboratory (JPL), uma instituição associada à NASA. O interesse destas instituições internacionais era o cálculo das órbitas precisas GPS, realizado pelo International GNSS Service (IGS), pois era necessária uma distribuição homogênea de estações no mundo todo. Somente no final de 1996, com a aquisição de sete equipamentos através do Fundo Nacional do Meio Ambiente (FNMA) em colaboração com a Escola Politécnica da Universidade de São Paulo (EPUSP), é que foi estabelecida a primeira estação da RBMC em Curitiba, a estação PARA. 
A RBMC é formada por um conjunto de estações com receptores GNSS que operam 24 horas por dia, durante os sete dias da semana, cujos dados GNSS são publicados diariamente no portal do IBGE. Em todas as estações são coletadas observações de código e fase (L1, L2 e em algumas L5), a um intervalo de coleta de 15 segundos, sendo sua disponibilização efetuada em arquivos diários nos formatos RINEX2 e RINEX3 juntamente com as órbitas operacionais. Ela foi a primeira rede geodésica ativa estabelecida na América do Sul, com o objetivo de materializar a estrutura de referência geodésica no Brasil e servir de ligação com as redes geodésicas internacionais. Na última década, através de parcerias com o Instituto Nacional de Colonização e Reforma Agrária (INCRA), Instituto Nacional de Pesquisas Espaciais (INPE) e dezenas de instituições públicas que colaboram na operação, a RBMC passou por um intenso processo de expansão e meIhorias, dentre as quais destaca-se a atualização dos equipamentos contemplando além do GPS (sistema americano) e GLONASS (sistema russo), os sistemas Galileo (europeu) e BeiDou (chinês).

Das 148 estações que compõem atualmente a RBMC, 121 operam em tempo real, constituindo o que se denomina serviço RBMC-IP. Nestas estações, as observações coletadas a cada segundo são transmitidas em tempo real para o centro da rede no Rio de Janeiro e disponibilizadas imediatamente na Internet no formato RTCM 3.0 e RTCM MSM (Multi Signal Messages), usando-se o protocolo TCP/IP denominado NTRIP (Networked Transport of RTCM via Internet Protocol), desenvolvido pela Agência Alemã de Geodésia e Cartografia. Através do serviço RBMC-IP os usuários, com acesso à Internet no campo, realizam levantamentos de precisão centimétrica em tempo real, seja no modo estático quanto no modo cinemático. O software ntripcaster, utilizado na distribuição dos dados e correções na Internet, foi disponibilizado ao IBGE por esta agência alemã com base na cooperação com o Serviço IGS em Tempo Real (IGS-RTS). De acordo com esta cooperação, os fluxos de dados em tempo real das estações da RBMC são liberados ao IGS para contribuírem no cálculo das órbitas precisas em tempo real, entre outros produtos IGS-RTS.

Além do uso intenso destes dados em aplicações práticas de georreferenciamento de imóveis, cadastro, posicionamento estático, navegação, etc., a RBMC também é um laboratório de pesquisas em diversas áreas, dentre as quais se destacam a |Geodinâmica e Meteorologia. Através de suas observações foi constatado que a crosta terrestre na região Amazônica possui um movimento vertical anual de amplitude aproximada de $9 \mathrm{~cm}$, decorrente da variação de massa d'água dos rios. Descobriu-se também que o Brasil é afetado por uma atividade ionosférica com altos níveis de oscilação, o que vem a ocasionar problemas na propagação das ondas eletromagnéticas na região do país.

Outra aplicação importante da RBMC consiste no monitoramento vertical de sítios onde estão instaladas estações da Rede Maregráfica Permanente para Geodésia (RMPG). Esta rede ativa implantada, mantida e operada pelo IBGE mede continuamente o nível do mar na costa brasileira nas localidades de Arraial do Cabo (RJ), Belém (PA), Fortaleza (CE), Imbituba (SC), Salvador (BA) e Santana (AP). Com a finalidade inicial de subsidiar o monitoramento do nível do mar ao longo da costa para fins de estabelecimento e monitoramento da origem dos valores de altitude ortométrica no país, ou seja, do referencial altimétrico, reveste-se hoje de uma importância ainda maior por conta da necessidade de acompanhamento das alterações do nível médio do mar provocadas pelo aquecimento global. A colocação de estações RBMC em sítios de estações mare- 
gráficas permite que os movimentos verticais da crosta terrestre em cada local sejam compensados nos registros maregráficos, caracterizando precisamente as alterações que se originam exclusivamente das mudanças do nível médio do mar.

A RBMC também subsidia as seguintes pesquisas científicas:

- $\quad$ Estudo e modelagem do clima espacial: a partir das observações de dupla frequência coletadas nas estações da RBMC, a área de Estudo e Monitoramento Brasileiro do Clima Espacial (EMBRACE) do Instituto Nacional de Pesquisas Espaciais (INPE) produz mapas que retratam o conteúdo total de elétrons na ionosfera, informação relevante tanto para correção das observações GNSS de uma frequência quanto para avaliação dos impactos da ionosfera na propagação das ondas eletromagnéticas em geral;

- Modelos numéricos de previsão do tempo: considerando que os dados coletados em tempo real pela RBMC permitem a determinação do conteúdo de vapor d'água na troposfera, especialmente em 28 estações dotadas de coletores de dados meteorológicos, os mesmos alimentam modelos numéricos de tempo calculados pelo Centro de Previsão de Tempo e Estudos Climáticos (CPTEC) do INPE e utilizados na previsão diária do tempo;

- $\quad$ Atividades sismológicas no Brasil: as coordenadas das estações da RBMC, determinadas semanalmente, contribuem para a análise dos fenômenos sismológicos que ocorrem no território, complementando as informações fornecidas pela Rede Sismográfica do Brasil (RSBR). Estes estudos são realizados pela Universidade de São Paulo, juntamente com outras instituições brasileiras.

- Doze estações pertencem à rede IGS e seus dados são utilizados diretamente no cálculo das órbitas precisas e dos erros dos relógios dos satélites produzidos por este serviço;

- $\quad$ Quinze estações pertencem à rede IGS em tempo real (IGS-RTS) e seus dados são utilizados diretamente no cálculo das órbitas precisas e erros dos relógios dos satélites em tempo real;

- $\quad$ A nível continental, suas estações fazem parte da rede de estações GNSS de operação contínua do SIRGAS (SIRGAS-CON), composta por mais de 400 estações na América Latina. Essa rede SIRGAS-CON materializa a estrutura geodésica de referência SIRGAS, permitindo um contínuo monitoramento e acompanhamento do seu comportamento geodinâmico no continente.

Em virtude da participação da RBMC na rede SIRGAS-CON, as coordenadas de suas estações são determinadas toda semana por Centros Locais de Processamento SIRGAS (dentre os quais encontra-se a Coordenação de Geodésia - CGED), o que, em última análise, subsidia o cálculo de modelos de velocidade, ou seja, de $d \varphi / d t$ e $d \lambda / d t$ de qualquer ponto do território brasileiro ( $\varphi$ e $\lambda$ representam, respectivamente, a latitude e longitude geodésicas referidas ao SIRGAS, e $\mathrm{d} / \mathrm{dt}$ representa a variação destas grandezas no tempo), evidenciando um deslocamento médio de um pouco mais de $1 \mathrm{~cm} /$ ano para noroeste provocado pelo movimento da placa tectônica SulAmericana.

A manutenção e a operação da RBMC melhoraram nos últimos anos, com apenas $6 \%$ de interrupções por mês, aumentando assim a sua credibilidade com a comunidade usuária. Segundo as estatísticas de download dos dados, nos últimos 12 meses foram baixados em média pelos 
usuários 480 mil arquivos por mês. Quanto ao serviço em tempo real (RBMC-IP), cerca de 500 usuários foram cadastrados nos últimos 12 meses. Os dados das estações da região sul e sudeste são os mais procurados pelos usuários tanto na área de download do portal do IBGE para o pós-processamento, quanto no serviço em tempo real.

\section{A Evolução Tecnológica na Informação Geoespacial no Brasil e no Mundo}

Quando se pensa que há três décadas a obtenção da uma posição geográfica em qualquer lugar do mundo estava na mão de profissionais especializados, engenheiros e geógrafos, que precisavam de equipamentos sofisticados para realizarem medições que levavam dias ou meses, sem contar o tempo dedicado ao cálculo das coordenadas, a é surpreendente a constatação de que qualquer um de nós com um simples smartphone obtém hoje esta posição em poucos segundos.

Graças à evolução tecnológica, a comunidade envolvida na produção e uso da geoinformação no mundo tem aumentado significativamente nas últimas décadas, bem como a compreensão do valor dela nas tomadas de decisão em governos e empresas. A Internet, dispositivos móveis e a explosão de serviços baseados em localização permitem que todos em qualquer lugar no mundo obtenham facilmente informações relevantes para a produção de produtos e serviços, levando a sociedade de uma maneira geral a apreciar o valor da geoinformação.

Entretanto, o que a maioria das pessoas desconhece é que para fazer o GPS do smartphone informar corretamente a posição em um aplicativo é necessário que os dois (o GPS e a base cartográfica onde esta posição será representada) falem a mesma língua, ou seja, utilizem o mesmo sistema de coordenadas.

Este foi um grande desafio, pois todo acervo cartográfico no Brasil estava num referencial que não era aquele usado pelo GPS. A associação de informações em mapas com informações obtidas a partir do GPS era um trabalho que sempre envolvia a transformação de coordenadas e cálculo de parâmetros que correlacionavam as coordenadas obtidas pelo GPS com os sistemas de referência antigos da cartografia.

O resultado desta reflexão levou o IBGE a conduzir um processo de transição para a adoção de um novo sistema geodésico de referência, compatível com as técnicas de posicionamento por satélites.

\section{A Mudança para o SIRGAS2000}

O IBGE, como responsável pelo Sistema Geodésico Brasileiro e co-responsável pelo Sistema Cartográfico Nacional, iniciou o processo de migração das coordenadas geodésicas no Brasil para um sistema compatível com as novas tecnologias, no caso o GNSS, há mais de 10 anos. 
Em fevereiro de 2005, através da Resolução da Presidência n 1/2005, o IBGE deu início ao processo de adoção do SIRGAS, tendo em vista que esse referencial tem compatibilidade com os novos sistemas de posicionamento, resolvendo problemas no emprego de informações e aplicações geoespaciais. Nesta Resolução foi previsto um período de transição de dez anos de convivência com os referenciais existentes. Durante este processo no país, foram disponibilizadas coordenadas em SIRGAS2000 - época 2000,4, aplicativos e serviços através dos quais os usuários podem obter coordenadas compatíveis com o GNSS.

Este período de transição foi finalizado no dia 25/02/2015 quando a Resolução da Presidência $n^{\circ} 1 / 2015$ definiu o seu término, orientando assim que todos os produtores de informações geoespaciais do país passassem a adotar exclusivamente no país o Sistema de Referência SIRGAS2000. Neste contexto, a RBMC atua como a estrutura geodésica de referência do SIRGAS2000, possibilitando também o acompanhamento da sua materialização ao longo do tempo.

\section{Serviço IBGE-PPP - Provendo o acesso ao SGB em SIRGAS2000}

Considerando que, na missão de gestor do SGB o IBGE necessita prover o acesso do usuário ao referencial oficial do país, as redes geodésicas ativas e passivas viriam suprir as demandas de dotar o país de estruturas geodésicas compatíveis com o nível de precisão proporcionado pela tecnologia atual, visando dar suporte às diversas atividades que necessitam de informações posicionais. Entretanto, através de levantamentos realizados recentemente foi identificado um elevado índice de destruição das estações pertencentes às redes passivas, mesmo daquelas estações estabelecidas em locais seguros e de configuração robusta (pilares de concreto).

Com a crescente evolução das geotecnologias, principalmente quanto ao posicionamento por satélites, identificou-se a necessidade de se disponibilizar um serviço online de processamento de dados GNSS que atendesse aos mais variados tipos de usuários que não possuem software de processamento. A partir dessa demanda e acompanhando o estado da arte das tecnologias de posicionamento, em abril de 2009 o IBGE disponibilizou o serviço denominado IBGE-PPP (Posicionamento por Ponto Preciso), sendo o primeiro país da América Latina a fornecer este tipo de serviço online. A partir desse momento, o IBGE trouxe aos usuários de suas informações geodésicas uma nova forma de prover o acesso ao SGB dissociado das redes geodésicas.

Durante estes 10 anos de operação do serviço no portal do IBGE foram realizadas algumas melhorias, como a redução no tempo de resposta dos processamentos, entre outras implementadas no relatório dos resultados. 
O crescimento mensal quanto ao uso do serviço é acompanhado de estatísticas, as quais comprovam o seu sucesso ao longo dos 10 anos de operação, bem como a credibilidade nos resultados obtidos em SIRGAS2000. Atualmente são contabilizados em média 1000 processamentos por dia, o que vem a constatar o sucesso deste serviço perante os usuários.

\section{A Visão da evolução do posicionamento no mundo}

Em 2011, foi criado o Comitê das Nações Unidas de Especialistas na Gestão de Informação Geoespacial Global (UN-GGIM) para garantir que os Estados-Membros possam trabalhar juntos, compartilhar conhecimentos e apoiar o desenvolvimento das informações geoespaciais. O trabalho do GGIM é baseado no reconhecimento do valor que a informação geoespacial pode desempenhar no desenvolvimento de nossas economias, prestação de serviços de emergência, desenvolvimento sustentável e, ao fazê-lo, melhorando a vida das pessoas em todo o mundo. O GGIM visa proporcionar uma coordenação entre os Estados-Membros e organizações internacionais, sobre o trabalho associado à gestão de informação geoespacial global.

No contexto deste Comitê, foi criado um grupo de trabalho que estuda as tendências das informações geoespaciais. Este grupo concluiu que nos próximos anos ocorrerá um aumento significativo no número de dispositivos associados com o GNSS, e suas funcionalidades, em conexão com a Internet e que, como resultado, se usará e criará cada vez mais informações de localização.

Ao considerar essas questões, em agosto de 2014 o UN-GGIM e o Conselho Econômico e Social das Nações Unidas (ECOSOC) durante sessão em novembro de 2014, propuseram adotar um sistema de referência geodésico mundial preciso, consistente, robusto e homogêneo para georreferenciamento e uma forte base metrológica para observações da Terra, bem como o acompanhamento das alterações globais e informações relacionadas.

O GGIM reconheceu a importância da cooperação internacional para implementar o referencial geodésico global e dos serviços para apoiar a tecnologia GNSS fornecendo a infraestrutura para todas as atividades geoespaciais, como um elemento essencial da interoperabilidade de dados espaciais, mitigação de desastres e desenvolvimento sustentável. Reconheceu também a importância econômica e científica para a construção de uma infraestrutura geodésica mundial precisa e estável para a Terra, permitindo a inter-relação de medições efetuadas em qualquer lugar da Terra e do espaço, usadas em diversas aplicações científicas e sociais, dentre as quais destacam-se: questões relacionadas com o nível do mar, acompanhamento das alterações climáticas, mitigação de desastres naturais e gestão dos riscos associados. 
Nesse sentido, foi aprovada na Assembleia Geral da ONU em 2015 a primeira resolução de sua história na área da informação geoespacial. Esta resolução, co-patrocinada por 52 Estados-membros, dentre os quais o Brasil, trata do tema "Infraestrutura Geodésica Global de Referência para o Desenvolvimento Sustentável", onde é reconhecida a importância de uma abordagem global coordenada para a Geodésia. Além disso, propõe uma cooperação multilateral na área, incluindo o compartilhamento de dados geoespaciais, a construção de capacidades nos países em desenvolvimento e a criação de normas e convenções internacionais. No caso do Brasil, esta iniciativa pavimenta de forma ainda mais sólida a estrada a ser seguida pela RBMC, considerando a sua integração continental com o SIRGAS e suas aplicações para o desenvolvimento sustentável. 